\section{Ephemeral Architectures: \\ the union of concepts in the creation of an immersive experience}

\author{
Paula Arzillo ${ }^{a}$ \\ Marcus Vinicius Pereirab
}

Universidade Anhembi Morumbi

This theoretical-methodological research aims a final graduation paper in the Architectures and Urbanism bachelor's degree program, which corresponds to an activity of synthesis and integration of skills developed throughout the course. Therefore, it is proposed to create a Project of an ephemeral architecture, which explores the perception and sensitivity of visitors, through the interactive experiences given by it.

The theoretical basis searches architectural practices that relate innovative Technologies, and which materialize through an immersive exhibition, which explores interactivity with the visitors, in order to stimulate human senses - such as touch, smell, taste and hearing. For this, it is taken advantage of organic shape study, relating the concepts of biomimetic design and parametric design in order to demystify complex formulas through computer generated algorithms.

The evolution process of the new digital tools brings an approximation of graphical representation with the real world. The complexity of the architectural technical representation, reached a parameter that becomes impossible without the computer software. In this context, parametric architecture is a technique developed from software, which has the ability to perform complex calculations, in a clear and effective way, allowing the creation of complex shapes that allow to expand the creative process until then, limited by technical characteristics.

The Project called "Feelings, an immersive experience is made up of six distinct spaces, divides by rooms for temporary exhibitions at the Pinacoteca do Estado de São Paulo. The beginning of the Project route is given by the central octagon, where is the first installation that has a large scale, inspired by a spider web materialized through tensioned lycra fabric. The others rooms are based on the shape and characteristics of animals: 1) the Octopus tentacles, which stimulate the human touch, through holes in the Octopus suckers, that invite visitors to find out different textures; 2) butterfly cocoons shapes, containing aromas capsules, which stimulate the sense of smell; 3) silhouettes of marine jellyfish shelter the dripping system of a surprise flavor drop, which awaken the taste buds, sensory receptors of the tongue as sweet, bitter, salty, sours or umami; 4) the chameleon's scaly skin, due to the ability to change colors, is taken to produce an interactive panel of lights that stimulates the sense of vision; 5) the structure of bird s taken to address the sense of hearing, by hiding speakers with sound effects that change according to the visitors interaction. All the structuring of these models used practices of digital architecture technologies.

Finally, this research applies practical solutions based on three-dimensional graphic models for the representation of projects with complex shapes through intensive use of modeling, standing out for uniting concepts such as biomimetics and parametric in favor of the practice of innovative tools and still not so much explored in the Architecture and Urbanism course, promoting future research and expanding the repertoire within the area.

KEYWORDS

Biomimetic, Experience, Exhibition, Interaction, Parametric Design 